\title{
Information-theoretic preprocessing method for computer vision systems
}

\author{
Tananykina L.V. \\ Research Institute of Applied Problems, Saint-Petersburg
}

\begin{abstract}
The aim of the research is finding an image obtaining method which is invariant to shooting conditions for further application of correlationextremal matching method in technical vision systems. The method based on entropy analysis is offered. Some testings of the method were carried out; images obtained in different conditions were used. The tests showed that preprocessed images have more stable correlation coefficient than original images.
\end{abstract}

Keywords: image preprocessing, technical vision, correlation systems, entropy analysis

Citation: Tananykina L.V. Information-theoretic preprocessing method for computer vision systems. Proceedings of Information Technology and Nanotechnology (ITNT-2015), CEUR Workshop Proceedings, 2015; 1490: 298-303. DOI: 10.18287/1613-0073-2015-1490-298-303

\section{Introduction}

Preprocessing methods are commonly used in present-day data analysis techniques. The main task in computer vision systems is obtaining images invariant to the capturing conditions or the physical nature of the sensor.

Main features which determine image internal structure are: bright points, lines, object edges, regions. In general they may be defined by brightness, color or texture contrast; and the direction (gradient) of the brightness difference (dark/light object on light/dark background) or of the color difference is also a-priori undefined.

It is difficult to use recognition procedures like correlation detector in case when images were captured at dissimilar conditions. But methods based on structural analysis are also insolvent in that case.

The information-theoretic image preprocessing method is offered for solving problems of comparison between images which differ in type of presentation or capturing conditions. The method effectiveness is demonstrated.

\section{Image correlation analysis. Formulation of the problem}

One of the typical objectives in technical vision is element-wise matching of two images of one object which were captured by different sensors or images captured by the same sensor but under different conditions or at different time. To make such a 
comparison we should do mutual binding of the images and compensate shifts, rotations, geometric and brightness distortions etc.

The classic way of finding superimposition of the pair of functions (to match pair of functions) is to find meaning of value measured correlation between these functions, and find position of the maximum of the correlation function.

For example, we need to match aerial photograph and topographic map of the same area (figure 1).

a)

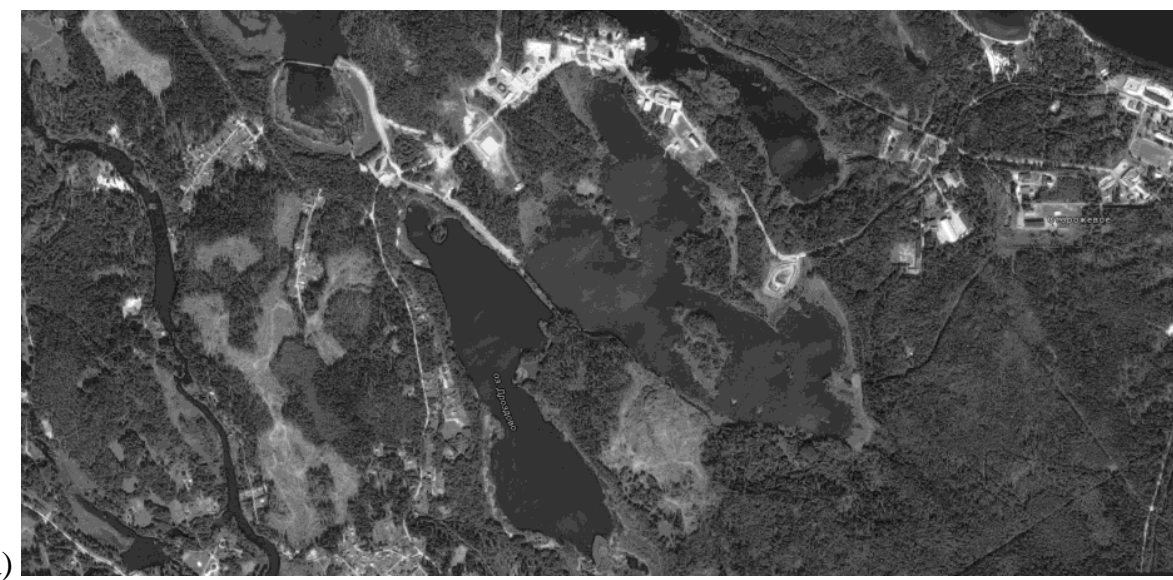

b)

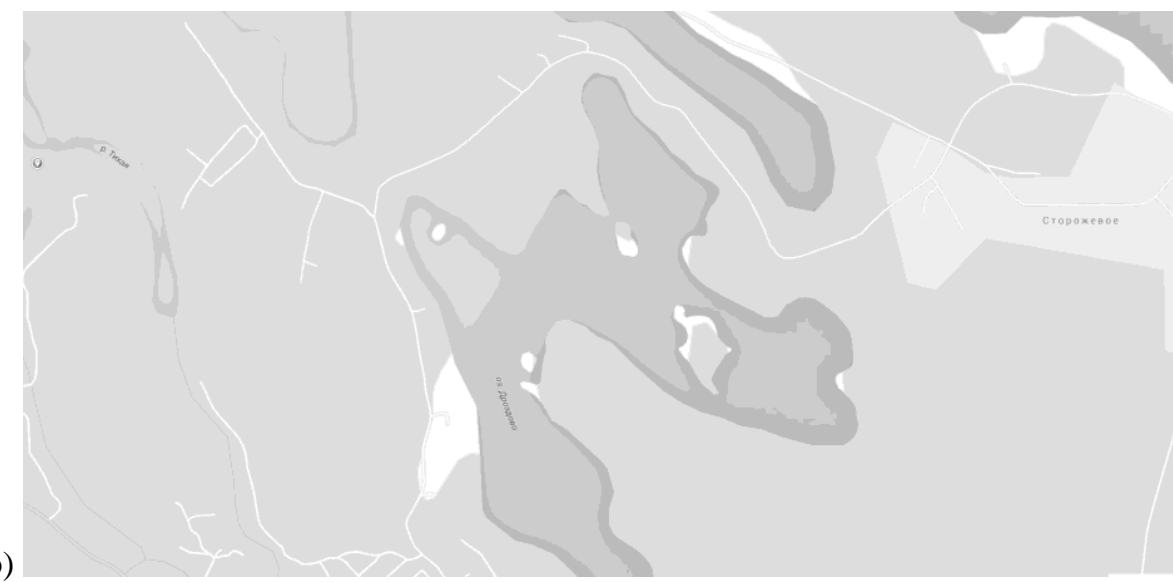

Fig. 1. - Images: a) aerial photograph; b) topographic map

Figure 2 shows the normalized cross-correlation function of the images.

The figure shows that the function has several peaks (two equal-amplitude maxima). The positions of both peaks are different from zero; although in this example images have zero shift. This example shows that the correlation-extreme method has serious limitations on the types of the compared images. 
a)

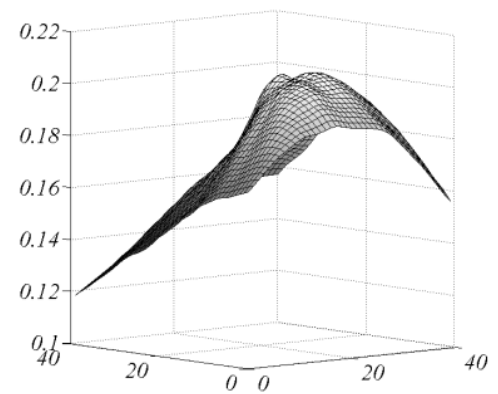

Fig. 2. - Normalized cross-correlation function of the images from fig.1

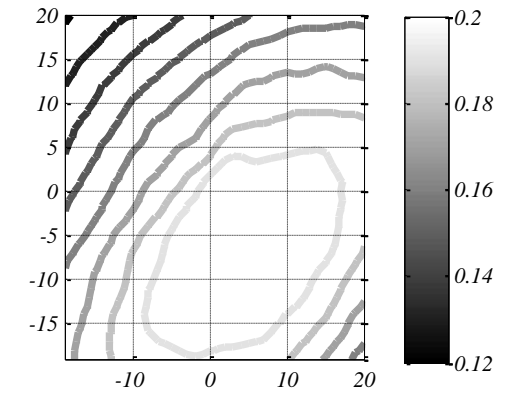

b)

As usual, methods of structural analysis are used for image matching. For example, specific points are detected and compared. But even for shifted images of the same type specific points can be different. Consequently, some special points may not have a pair, or the specific points in the pair will have a slightly different spatial position. In the case of dissimilar images special points will be unique for each image.

There is one thing that we should take into consideration: correlation-extremal method gives more precise solution than structural methods. Besides, there are effective algorithms and hardware for image correlation analysis which are already used.

That's why it is of great interest to adapt correlation-extremal method for matching dissimilar images. It's offered to use preprocessing method based on entropy analysis. To be more correct it is offered to substitute image with its' local entropy map, because local entropy map is more resistant to the effects of the factors we studied (as will be shown below).

\section{Image preprocessing method based on entropy analysis}

The solution of the problem is achieved by using the method of image preprocessing based on by taking into account existing internal statistical relationships between image elements. This decision is based on the assumption that statistically interconnected elements in a changing conditions of image capturing are statistically related.

Information-theoretic methods are used for identification of internal statistical relations in any type of data. To adapt correlation-extremal method for matching dissimilar images it is proposed to do entropy analysis of the images, namely to calculate local entropy maps of the images.

The image is considered as a realization of random process. Local entropy $\left(H_{i}\right)$ characterizes the degree of surprise happening of i-th event (occurrence). The less its' a-priori probability is, the greater its' local entropy.

Let's define what is implied under "event". An event $(e)$ is a specific brightness of the pixel. To calculate the probability of event e $(p(e))$ we should count the number of 
occurrences of the combination over the defined area $M \times N$ (or entire image) and divide by the total number of considered pixels.

The output image is formed by replacing each pixel with value, calculated according to the formula of local entropy (i.e. local entropy map is calculated):

$Y(i, j)=-\sum_{m=-(M-1) / 2}^{(M-1) / 2} \sum_{n=-(N-1) / 2}^{(N-1) / 2} p\left(e_{i+m, j+n}\right) \cdot \log \left(p\left(e_{i+m, j+n}\right)\right)$.

Figure 3 shows local entropy maps of images from fig.1.

a)

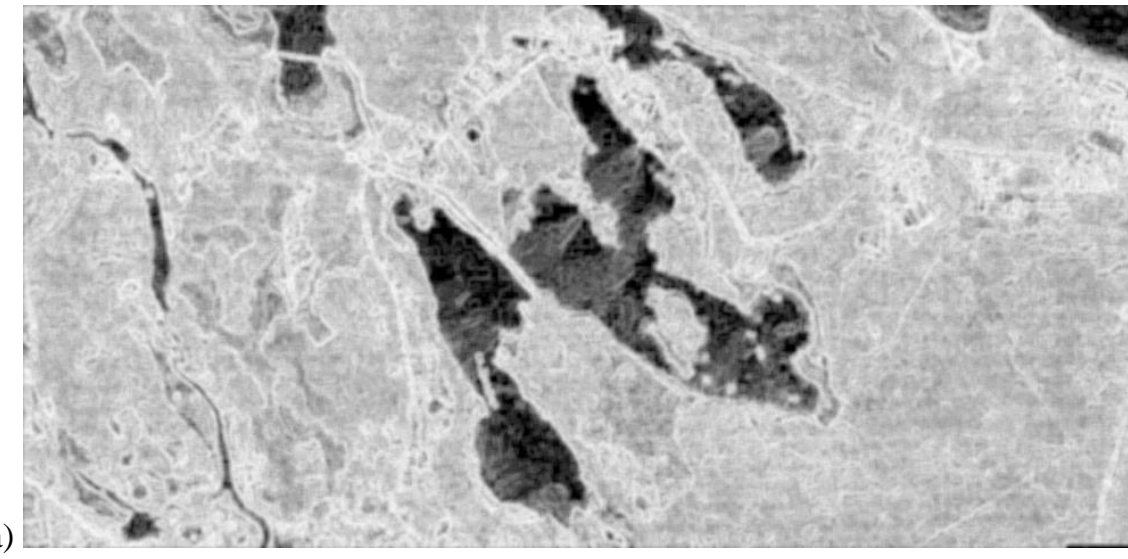

b)

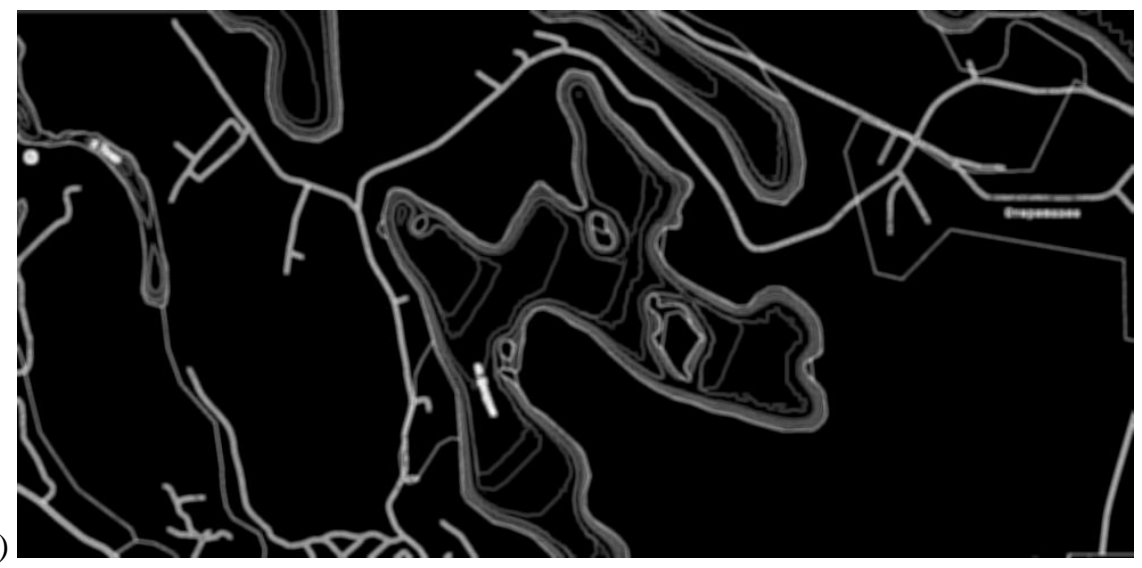

Fig. 3. - Local entropy maps: a) photo; b) map

Figure 4 shows normalized cross-correlation function of the local entropy. As we can see from the figure, the correlation function has a strongly marked maximum corresponding to zero shift. 


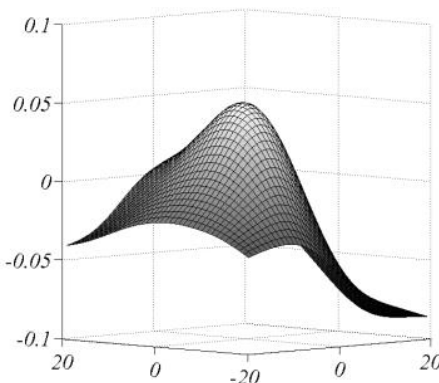

Fig. 4. - Normalized cross-correlation function of the images from fig.3

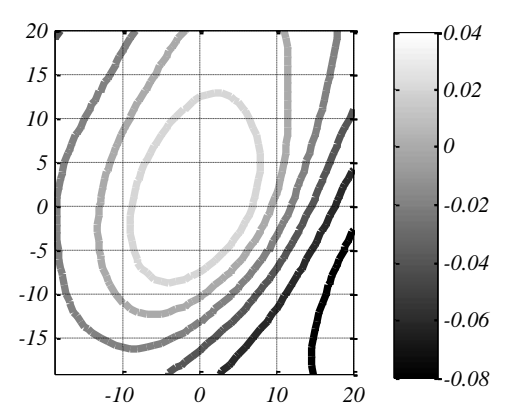

b)

Similar results were obtained using other test images: maps and aerospace photographs, photographs in winter and in summer, optical and infrared photographs. Test images after preprocessing demonstrate greater correlation coefficient and smoother correlation function than original images.

Thus, the entropy preprocessing method can be recommended for application in the correlation-extreme image matching for two reasons:

- it increases the correlation between dissimilar images;

- correlation function of entropy maps is smoother than the correlation function of the original images, whereby it is possible to significantly reduce the amount of computation to find the extremum.

\section{Conclusion}

The advantages of the correlation analysis are: this technology has been worked out many times before; there are fast algorithms to calculate correlation and hardware-based solutions. Currently it is of great interest to develop simple and cheap correlation vision systems implemented as special processors.

The aim of this paper was to use correlation method for image matching but in difficult conditions. Information-theoretic preprocessing technology allows comparing images more effective and correct. Researches of the method based on local entropy have shown that dissimilar images of the same scene have greater correlation coefficient after preprocessing. Developed method allows matching the following types of images automatically:

- aerial photographs captured at different times;

- aerial photographs and topographic map;

- optical and infrared images.

Thus, the application of developed method will significantly expand the application area of the correlation-extreme image matching method in computer vision systems. 


\section{References}

1. Tsvetkov OV, Polivanayte LV, Kutsenko SA, Repina MV. Simple highly informative image quality metrics in biomedical systems. Biotechnosphere, 2014; 1-2(31-32): 55-57.

2. Syrjamkin VI. Correlation-extremal radionavigation systems. Tomsk: Tomsky University Publisher, 2010.

3. Pratt WK. Digital image processing. New York: Wiley-Interscience, 1982.

4. Kullback S. Information theory and statistics. Gloucester: PeterSmith, 1978. 